\title{
특별부록
}

\section{Preparation and Characterization of Liposomes Containing Green Tea and Roselle Extracts to be Used in Cosmetics}

\author{
Bosompemaa Gyamera (Institute of Industrial Research-CSIR, Ghana) \\ Young-Ho Kim (Hankyong National University, Korea)
}

\begin{abstract}
Liposomal formulations have been used in the treatment of dermatological diseases with synthetic and herbal drugs incorporated to improve efficacy while herbal extracts reduce side effects associated with incorporated synthetic drugs.

Anti-oxidants in Green Tea leaves and Roselle calyces were extracted into liposomes containing cholesterol by reverse phase evaporation. Varying molar ratios of Lecithin, Tween 80, Cholesterol at varying concentrations were analyzed and compared.

The yield of the Green tea leaves water extracts (GTLW) and total Phenolic content (TPC) were found to be (30.35 $\pm 0.90 \%, 325.13 \pm 1.04 \mathrm{mgGAE} / \mathrm{g})$, Green tea leaves powder water extracts (GTPW) were found to be $(27.83 \pm$ $1.38 \%, 272.65 \pm 0.15 \mathrm{mgGAE} / \mathrm{g}$ ). Roselle calyces water extracts (GHFW) were
\end{abstract}

Author: Bosompemaa Gyamera (Principal Technologist, Institute of Industrial Research-CSIR, Ghana) (krispalmco@yahoo.com)

Corresponding author: Young-Ho Kim (Professor, Chemical Engineering Department of Hankyong National University, Korea) (ynhkim@hknu.ac.kr)

(c) Copyright Korea International Cooperation Agency. This is an Open-Access article distributed under the terms of the Creative Commons Attribution Non-Commercial License (http://creativecommons.org/ licenses/by-nc/4.0/) which permits unrestricted non-commercial use, distribution, and reproduction in any medium, provided the original work is properly cited. 


\section{Abstract}

found to be (52.42 $\pm 3.23 \%, 37.82 \pm 0.21 \mathrm{mgGAE} / \mathrm{g})$, Roselle calyces powder water extracts (GHPW) were found to be $(51.49 \pm 1.30 \%, 50.00 \pm 0.11 \mathrm{mgGAE} / \mathrm{g})$. In the combination of water and organic solvent, the Green tea leaves extracts (GTLWC) were found to be $(19.22 \pm 1.20 \%, 246.67 \pm 0.17 \mathrm{mgGAE} / \mathrm{g})$, Green tea leaves powdered extracts (GTPWC) were found to be $(19.42 \pm 2.28 \%, 180.16 \pm$ $0.81 \mathrm{mgGAE} / \mathrm{g}$ ), Roselle calyces extracts (GHFWC) were found to be $(52.42 \pm$ $3.23 \%, 37.82 \pm 0.21 \mathrm{mgGAE} / \mathrm{g}$ ), Roselle calyces powdered (GHPWC) extracts were found to be $(56.06 \pm 1.9 \%, 38.52 \pm 0.34 \mathrm{mgGAE} / \mathrm{g})$.

The ethanol extracts (GTLE \& GTPE) are greenish with \% average yield of $33.66 \pm 0.23 \& 34.86 \pm 3.17$. The water extracts (GTPW \& GTLW) are light green in appearance with \% average yield of $27.83 \pm 1.38 \& 30.35 \pm 0.9$. Ethyl acetate \& chloroform (GTLWC \& GTPWC) extracts shows brownish green in appearance with \% average yield of $19.22 \pm 1.20 \& 19.42 \pm 2.28$ respectively.

Phase contrast microscope (PCM) results show that ACT80E has particle size (PS) of (47.655 $\pm 10.32 \mu \mathrm{m})$. Liposomes containing only cholesterol with Roselle extract (ACT80HB), with PS (54.588 \pm 10.20$)$. Liposomes containing only cholesterol with green tea extract (ACT80GT) is the highest with PS (69.668 \pm 19.92).

It was observed that the mean particle size of the green tea and Roselle extract loaded liposomes were higher than the unloaded liposomes' mean particle size. The higher mean particle size of the extract loaded liposomes result might be explained by the fact that phenolic compounds might be incorporated into a lipid bilayer and/or may be absorbed onto the surface of liposomes as well as incorporated into the interior region of the liposomes which could be due to hydrogen bonding between polar head groups and the phenolic compounds in the extract, hydrophobic interactions between the fatty acid tail so of the polar lipid and the more hydrophobic moieties of the phenolic compounds, or thermodynamic driving forces such as the liposomes attaining a more optimal configuration (Gibis et al., 2012).

주제어: Liposome, Reverse Phase Evaporation, Natural Antioxidant (Green Tea and Roselle), Cholesterol, Squalane 


\section{INTRODUCTION}

\section{Necessity of This Study}

In recent times, people are more concerned about their health and appearance. There is therefore a high demand for products formulated with natural and nutraceutical ingredients. Innovative delivery systems are driving the new product development in the field of cosmetics (Patravale \& Mandawgade, 2008).1)

Today, consumers are looking for personal care products that supply multiple benefits with minima efforts. A significant number of novel products are based on a new generation of active ingredients. With these emerging actives, come a range of formulation challenges that include stability control and the complications of combining several actives into a single cosmetic product (Fakhravar et al., 2016; Patravale \& Mandawgade, 2008).

Liposomes have been extensively studied as drug carriers in the pharmaceutical and medical fields. Research has expanded considerably over the last 30 years, increasing applications area from drug and gene delivery to diagnostics, cosmetics (Fakhravar et al., 2016)2), food and chemical industry. The superiority of liposomes as drug/nutrient carriers has been widely recognized. Several reviews about liposomes as drug delivery systems and specific application via oral, topical, pulmonary, and ophthalmic route have been published.

\section{Background Information}

Liposomes are spherical lipid bilayer surrounding an aqueous core (Edwards, 2016). They are small vesicles composed of phospholipids (Verma et al., 2003). The lipid bilayer of liposomes is composed of double chain, amphiphilic lipids with their nonpolar, hydrophobic tails aligned with each other and their polar, hydrophilic headgroups oriented toward the exterior aqueous solution or directed

1) www.advanceddermatologypc.com

2) www.researchgate.net 
toward the inner aqueous core (Edwards, 2016). Liposomes can be categorized in different ways based on the diameter and the number of lipid bilayers, liposomes are often classified as small unilamellar vesicles SUVs, 20-100 nm), large unilamellar vesicles (LUVs, 100-1,000 nm), giant unilamellar vesicles (GUVs $>1,000 \mathrm{~nm}$, and multilamellar vesicles (MLVs > $500 \mathrm{~nm}$ ) (Zhang, 2017).

Liposomes can be negatively charged, neutral or positively charged by the addition of other materials (Zhang, 2017) as a certain pH environment (D'Souza, 2017).

Liposomes with a single lipid bilayer are termed unilamellar vesicles and their classification according to size is generally such that small unilamellar vesicles typically have sizes between 20 and $100 \mathrm{~nm}$; those with diameters between 100 $\mathrm{nm}$ and $1 \mu \mathrm{m}$ are generally termed large unilamellar vesicles; and liposomes above $1 \mu \mathrm{m}$ are termed giant unilamellar vesicle (Edwards, 2016).

Unilamellar liposomes comprise a single bilayer encapsulating an aqueous core. And multilamellar liposomes comprise multiple concentric bilayers with encapsulation possible both within the core and between adjacent bilayers (Edwards, 2016).

The liposomal bilayer is typically composed of a mixture of phospholipids and cholesterol. Phospholipids are amphiphilic molecules consisting of two long fatty acid tails forming a hydrophobic portion, which are linked via esters through glycerol to a phosphate group and hydrophilic headgroup (Edwards, 2016).

Cholesterol imparts reduced permeability to charged and water-soluble molecules by effectively filling in gaps (Edwards, 2016) and enhancing the rigidity of the liposomal bilayer (Lu, 2014).

Lipids have characteristics transition temperature (Tc) which represents the temperature at which they transform from a solid or "gel" phase to liquid or fluid phase. The temperature at which this transition occurs is dependent on the liquid or fluid phase. The temperature at which this transition occurs is dependent on the lipid chain length, degree of unsaturation, presence of chain side groups, as well as the structure of the hydrophilic headgroup. Transition temperature decreases with the degree of unsaturation or side groups in the hydrocarbon chain. Hydration of lipid mixtures above the transition tempe- 
rature of the composite lipids is necessary to afford a fluid state, which greatly facilitate manipulations required during preparation (Edwards, 2016).

Liposomes are prepared using one of the following methods as reverse phase evaporation, thin film hydration, ethanol/ether injection (Lu, 2014) freeze thaw, dehydration/rehydration (Sulkowski et al., 2005), sonication and detergent dialysis. The choice of method depends on the desired properties of the liposomes and the nature of the material to be encapsulated (Edwards, 2016). Liposomes are used for higher concentration of drugs in deeper layers of skin and a reduction in percutaneous absorption and unwanted side effects (Singh et al., 2014).

These are used as a good delivery vehicle for plant extract. Various extracts can be incorporated into it like turmeric, carrot extract (Singh et al., 2014), papaya extract, aloe-Vera etc. The incorporation of herbal extract into liposome reduces the side effects which are associated with the synthetic drugs (Singh et al., 2014).

The main object was to prepare and characterize liposomal formulations containing Green tea (Camellia sinensis) and Roselle (Hibiscus sabdariffa) calyces extracts as an antioxidant activity indicator and the influence of the extracts on liposome size to be used in cosmetics.

The specific objectives were:

1. To prepare extracts containing natural antioxidant from Green tea and Roselle.

2. To develop empty liposomes containing cholesterol.

3. To develop liposome containing green tea and roselle extracts.

4. To characterize, evaluate the stability of liposomes and compare results

\section{Antioxidants}

Antioxidants are defined as any substance that delays or inhibits oxidation of a substrate even at low concentrations (Sindhi et al., 2013).

\section{1) Green tea (Camelia sinensis)}

Green tea (Camellia sinensis) has become as one of the most important and 
commonly consumed herb due to health benefits associated with its high catechin content (Dag \& Oztop, 2017; Horie \& Kohata, 2000). The major chemical components of the green tea leaf that provides all of these health benefit are polyphenols. Main polyphenols in green tea include gallic acid, quercetin, kaempferol, myricetin and their glycosides (Babu et al., 2008; Dag \& Oztop, 2017; 〈Figure 1〉).

\section{2) Roselle (Hibiscus sabdariffa)}

Hibiscus sabdariffa L. is a shrub belonging to the family Malvaceae and is popularly known (Bergmeier et al., 2014; Daniele, 2014) as hibiscus or roselle. In botanical terms, it is a thick red plant with fleshly cup-shaped calyxes. The calyxes, rich in phenolic compounds with several physiological activities, also contain large amounts of pectin, anthocyanin, ascorbic acid, malate and protocatechuic acids which may have diuretic and choleretic effects (Bergmeier et al., 2014; Daniele, 2014).

In fact, they decrease blood viscosity, reduce blood pressure and stimulate intestinal peristalsis (Anokwuru et al., 2011; Bergmeier et al., 2014; Daniele, 2014). Roselle extracts have a brilliant red color and are used in the industrial manufacture of jellies, jams, preserves, sauces, beverages, besides being a good source of natural food colorants (ESSELEN; SAMMY, 1975).

Since Roselle extracts contain several polar and acidic compounds, their chemical properties have hampered their use in skin products due to skin irritation and low skin permeation (Hjorth, 1965; Pinsuwan et al., 2010; 〈Figure 2〉).

\section{〈Figure 1〉 Green tea plantation (a) green tea leaves (b)}

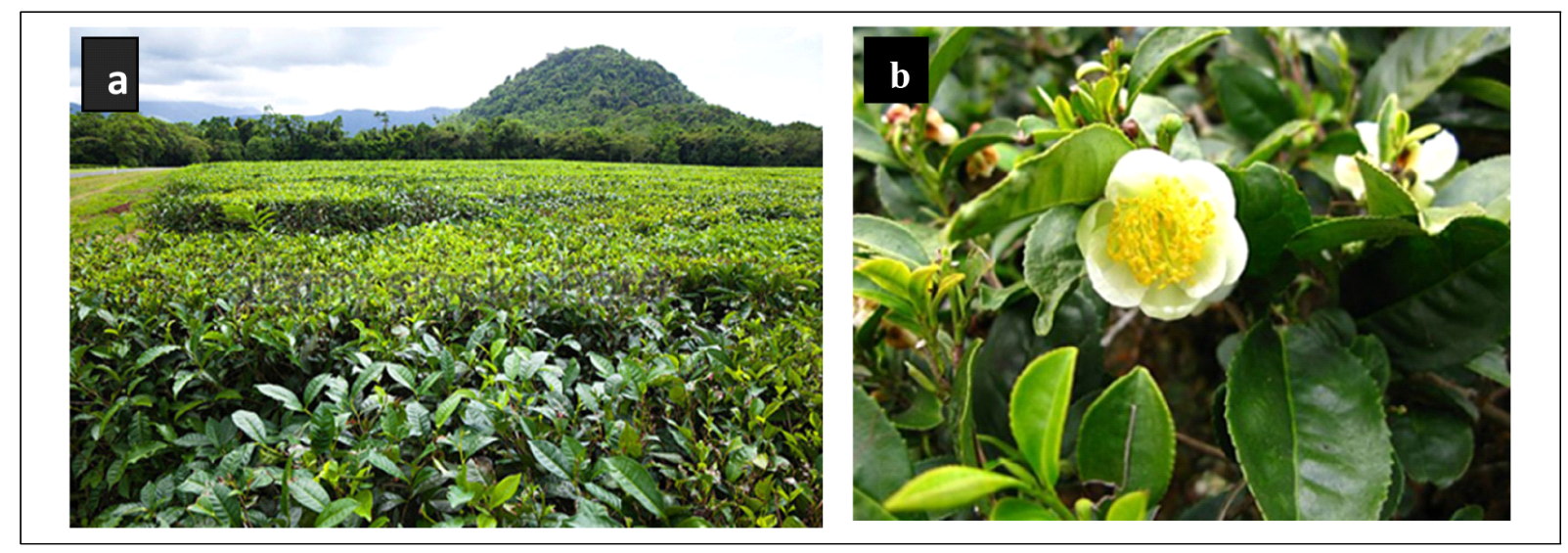


〈Figure 2〉 Roselle plantation (a) and Roselle leaves (b) fresh Roselle calyces (c) and dried Roselle calyces (d)

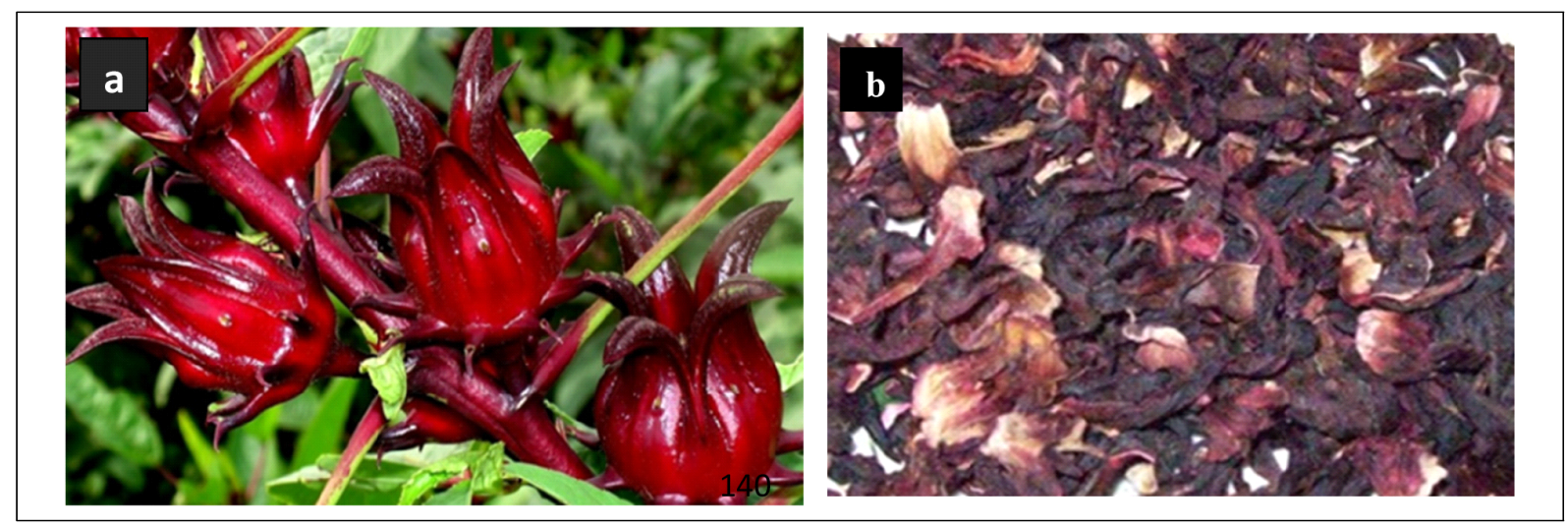

\section{Lipids}

Animal fats and vegetable oils mainly consist of triglycerides that are of glycerin, a trivalent alcohol which is combined with three fatty acids (esterified). When speaking of lipids in today's cosmetic products the term lipid generally is extended to other lipophilic substances like waxes and mineral oils.

\section{Concept of Liposome}

A liposome is a tiny bubble (vesicle), made out of the same material as a cell membrane (Choudhury, 2013; Dua et al., 2012). Structurally, liposomes are concentric bleeder vesicles in which an aqueous volume is entirely enclosed by a membranous lipid bilayer. Membranes are usually made of phospholipids, which are molecules that have a hydrophilic head group and a hydrophobic tail group (Dua et al., 2012).

A liposome is a spherical vesicle having at least one lipid bilayer (〈Figure 3〉). The liposome can be used as a vehicle for administration of nutrients and pharmaceutical drugs. Liposomes can be prepared by disrupting biological membranes (such as by sonication).

Liposomes are used as carriers for numerous molecules in cosmetics, pharmaceuticals, food and farming industries. These industries employ the use of liposome encapsulation to grow delivery systems that can entrap and shield the 

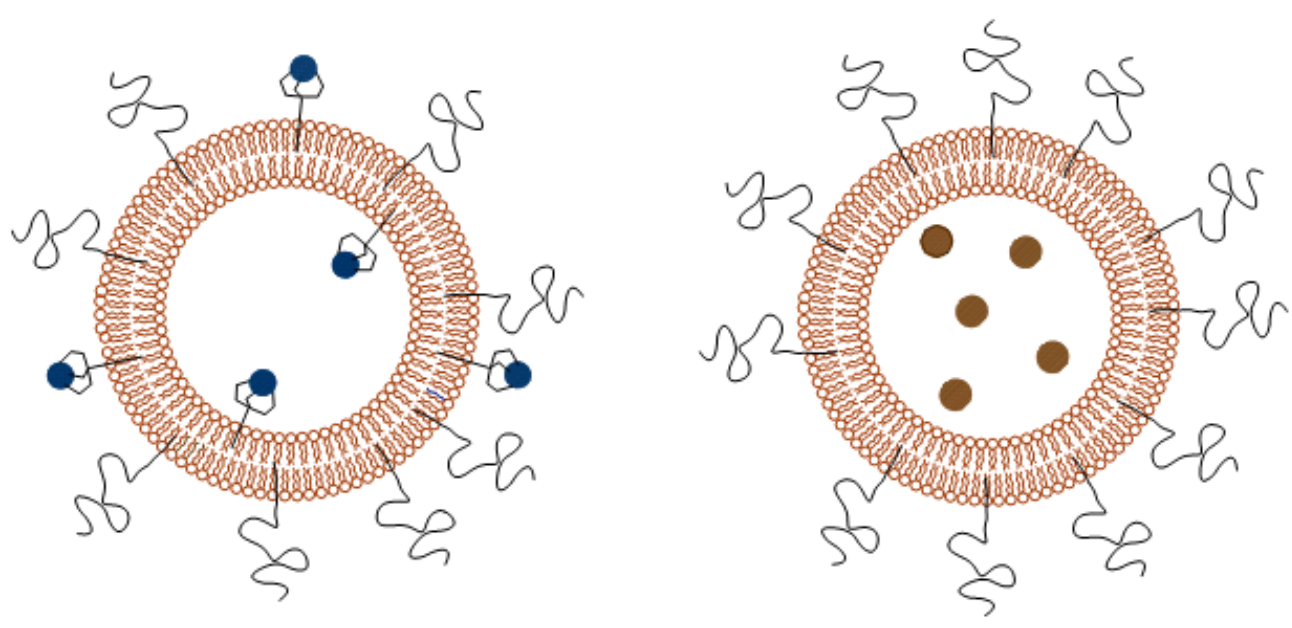

functionalities of unstable compounds such as antimicrobials, antioxidants, flavors and bioactive elements (Akbarzadeh et al., 2013). Liposomes carry cosmetic agents better into the horny layer and the conditioning effect causes the horny layer to become a depot for these agents (Dragicevic \& Maibach, 2016; Lautenschlager). Liposome properties differ considerably with lipid composition, surface charge, size, and the method of preparation (Tejeda-Mansir et al., 2019). Furthermore, the choice of bilayer components determines the 'rigidity' or 'fluidity' and the charge of the bilayer (Akbarzadeh et al., 2013; Grumezescu, 2018).

The amphiphilic nature of phosphatidylcholine allows them to self-aggregate in an aqueous solution and to form their spherical structures. Liposomes can be unilamellar or multilarmellar and their sizes range from $50 \mathrm{~nm}$ to several $\mu \mathrm{m}$ depending on their method of production (New, 1990). They can deliver either hydrophilic (in the aqueous inner core) or lipophilic substances (in the lipid bilayer). Increased rates of skin permeability have been found for various active ingredients, e.g. progesterone and hydrocortisone, when they were applied topically in liposomal form. Also, less frequently side effects were observed when liposomal formulations were employed (Ho et al., 1985; Lasch \& Wohlrab, 1986). In contrast to these observations, other investigators could not find improved skin penetration of substances when they were applied in liposomes (Knepp et al., 1990). Also, liposomes themselves did not seem to penetrate through the sc in these early studies (Knepp et al., 1990). The conflicting results 
may stem from different lipid compositions of the liposomes employed as in addition to size. The lipid composition determines the physical characteristics of the liposomes and, therefore, also the interaction of these carrier systems with the skin (Choi \& Maibach, 2005). In the last few years some papers highlighted important factors that influence the penetration of active ingredients encapsulated in liposomes. Liquid-state, flexible liposomes showed greater skin penetration than those in a gel-state, small-sized and unilamellar vesicles seem to result in a higher degree of skin penetration (Blume et al., 2003; Verma et al., 2003). The application form can also influence the penetration kinetics (Cevc \& Blume, 1992).

\section{Methods of Liposome Preparation}

There are four basic stages in all the methods of liposome preparation (Akbarzadeh et al., 2013):
a. Drying down lipids from organic solvent.
b. Dispersing the lipid in aqueous media.
c. Purifying the resultant liposome.
d. Analyzing the final product.

There are two basic techniques for liposome preparation. These are;
a. Passive loading techniques.
b. Active loading techniques.

Active loading techniques involve three different methods such as

a. Mechanical dispersion method.

b. Solvent dispersion method and.

c. Detergent removal method (removal of non - encapsulated material) (Dua et al., 2012).

\section{Cosmetic Application}

The properties of liposomes can be utilized also in the delivery of ingredients 
in cosmetics. Liposomes offer advantages because lipids are well hydrated and can reduce the dryness of the skin which is a primary cause for ageing. Also, liposomes can supply replenish lipids and importantly linolenic acid to the skin. The first liposomal cosmetic product to appear on the market was the anti-ageing cream "Capture" launched by Christian Dior in 1986 (Muller-Goymann, 2004). Liposomes have been also used in the treatment of hair loss; minoxidil, a vasodilator, is in the active ingredient in products like "Regaine" that claim to prevent or slow hair loss (Lautenschlager, 2006). The skin care preparations with empty or moisture loaded liposome reduce the transdermal water loss and are suitable for the treatment of dry skin. They also enhance the supply of lipids and water to the stratum corneum (Patravale \& Mandawgade, 2008). Various liposome formulations were compared in vivo for cosmetics application (Betz et al., 2005) liposome formulations prepared from egg phospholipids exhibited a 1.5-fold increase in skin water content, whereas liposome formulations prepared from soya phospholipids showed no advantage compared to the references. Skin water content was measured daily and the results showed that skin humidity was increased significantly for the formulation containing 20\% egg phospholipids during 6 days. Since 1987, several cosmetic products have been commercially available; they range from simple liposome pastes which are used as a replacement for creams, gels and ointments to formulations containing various extracts, moisturizers, antibiotics, etc. Un-rinseable sunscreens, long lasting perfumes, hair conditioners, aftershaves, lipsticks, make-up and similar products are also gaining large fractions of the market (Laouini et al., 2012).

\section{Lipids Use in the Preparation of Liposome}

\section{1) Phospholipids (Lecithin)}

A phospholipid is a lipid that contains a phosphate group and is a major component of cell membranes. A phospholipid consists of a hydrophilic (waterloving) head and hydrophobic (water-fearing) tail. The phospholipid is essentially a triglyceride in which a fatty acid has been replaced by a phosphate group of some sort (〈Figure 4$\rangle)$. 


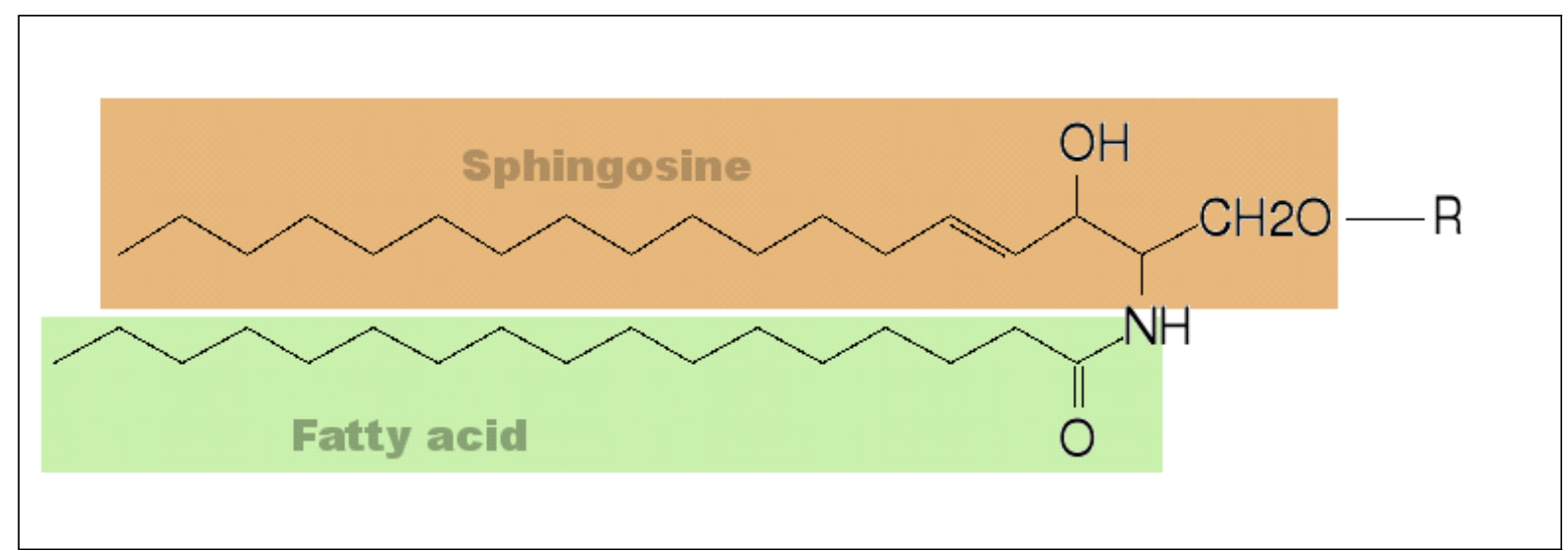

Source: Chemistry LibreTexts.

The liposomes are primarily composed of phospholipids although other lipids such as galactolipids might also be incorporated into the composition. The phospholipids which is the major components of biological membranes can be divided into two categories (glycerophospholipids and sphingomyelins) according to the alcohols contained in phospholipids.

Glycerophospholipids which are the main phospholipids found in eukaryotic cells contain the glycerol in their backbone. All natural glycerophospholipids are present in $\alpha$-structure and L-configuration. The head group, the length and the saturation of hydrophobic side chains, the type of bonding between the aliphatic moieties and glycerol backbone, and the number of aliphatic chains determine the chemical structure of glycerophospholipids. According to the head group type, glycerolphospholipids can be grouped as phosphatidylcholine, phosphatidylethanolamine, phosphatidylserine, phosphatidic acid, phosphatidylinositol, phosphatidylglycerol, cardiolipin (Li et al., 2015). In phosphatidylcholine, two acyl hydrocarbon chains are linked to glycerol at the sn-1 and sn-2 position by an ester linkage. The third glycerol carbon linked to a phosphate at the sn-3 position which is then linked to a choline group. The two hydrocarbon chains provide the hydrophobic tail and the hydrophilic phosphocholine provides the polar head group to phosphatidylcholine.

\section{2) Cholesterol}

A very important material in the liposomal structure that controls the stiffness 
is the content of cholesterol. Numerous studies on the use of cholesterol as a stabilizer has been conducted, showing that this steroid can increase the packing of phospholipid molecules improve vesicle resistance to aggregation, change the fluidity of intra-vesicle interactions to make them more rigid and sustain in severe shear stress of the lipid bilayer and reduce drug incorporation efficiency (Briuglia et al., 2015).

\section{3) The skin}

The skin is the largest organ of the body, with a total area of about 20 square feet. The skin protects us from microbes and the elements, helps regulate body temperature, and permits the sensations of touch, heat, and cold.

Skin has three layers:

- The epidermis, the outermost layer of skin, provides a waterproof barrier and creates our skin tone.

- The dermis, beneath the epidermis, contains tough connective tissue, hair follicles, and sweat glands.

- The deeper subcutaneous tissue (hypodermis) is made of fat and connective tissue.

The skin's color is created by special cells called melanocytes, which produce the pigment melanin. Melanocytes are located in the epidermis.

\section{4) Stability of liposome and shelf life}

The liposome stability is a major concern needed to be taken into consideration for the liposome formation, storage and delivery. Thus, the physical and chemical stability of the liposomes should be monitored by characterization of the liposome over a certain time period. Generally, the physical stability refers to the preservation of liposome structure characteristics while the chemical stability is related to the change in molecular structure of liposomes (Chrai et al., 2002). The zeta potential, average particle size, polydispersity index, encapsulation efficiency, lamellarity determination, phase and quantification of the residual solvent are the most common parameters used in the characterization of liposomes (Laouini et al., 2012). A detailed description of the methods used in the present study are given in the following sections. 


\section{5) Liposome observation}

Due to the fact that most particle size determination methods have difficulties to differentiate individual and aggregated liposome, microscopic observation of the liposome is required in order to confirm particle size measurement and to gain valuable information about the structure of the liposomes. Although light microscope provides several advantages of obtaining vesicle images in a short time using the standard laboratory equipment, information about the vesicle morphology, the sample heterogeneity regarding the shape and size, and differentiation of single and aggregated liposome it is inadequate to gain comprehensive information about the lipid bilayer of liposomes which is offered by other microscope techniques (Bibi et al., 2011).

Electron microscope offers greater magnification which allows to obtain information about lipid bilayer characteristics and the visualization of structure of much smaller unilamellar vesicles which cannot be visualized by light microscope. More specifically, while light microscopes have a resolution of 200 $\mathrm{nm}$, electron microscopes offer a resolution about $0.2 \mathrm{~nm}$. The common electron microscopes used in the characterization of liposome are transmission electron microscope which gives the information about internal structure of particles and scanning electron microscope indicating surface morphology of the particles. In the most basic terms, electron microscope working principle is based on the exposure of the sample to an electron beam which is focused by various lenses. Some electron then collide and displace electrons around the nuclei of atoms in sample while the rest of electron change their path only. Eventually, a projected image is created by focusing and magnifying electrons using a system of magnetic lenses (Bibi et al., 2011).

\section{MATERIALS AND METHODS}

\section{Materials}

\section{1) Plant materials}

The dried calyces of Hibiscus sabdariffa (Roselle) and Camellia sinensis (Green 
tea) were obtained from Madina market, La Nkwatanan district in the Greater Accra region in Ghana and SEO LIM Scientific systems in Korea respectively as shown in 〈Table 1$\rangle$.

Before extraction process, both samples were divided into two halves and ground using a household blender (〈Figure 5$\rangle$ ).

\section{2) Materials for lipid synthesis}

Phosphatidylcholine (Soybean Lecithin) and cholesterol were obtained from SEO LIM Scientific systems 〈Table 2〉, 〈Figure 5〉.

\section{〈Table 1〉 A table showing plant materials}

\begin{tabular}{c|c|c|c}
\hline Common name & Scientific name & Quantity & Producer/company \\
\hline Roselle & Hibiscus sabdariffa & $500 \mathrm{~g}$ & Purchased in Madina market from Ghana \\
\hline Green tea & Camellia sinensis & $500 \mathrm{~g}$ & Super Food Ltd. \\
\hline
\end{tabular}

〈Figure 5〉 Dried Roselle calyces (a) and dried green tea leaves (b)
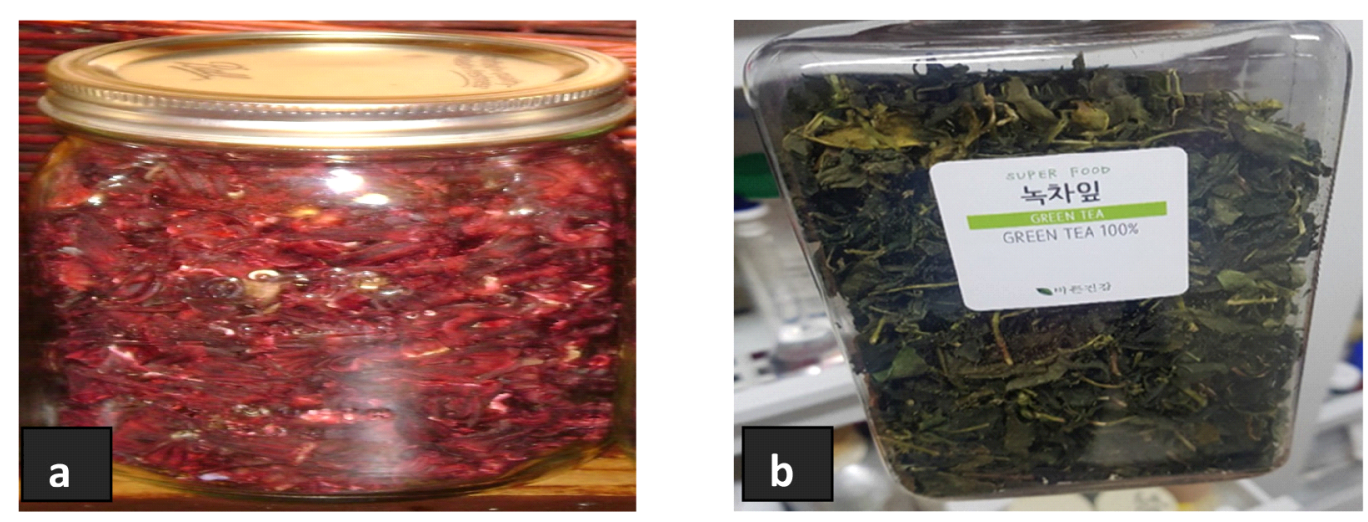

〈Table 2〉 A table showing lipids used in the formulation

\begin{tabular}{c|c|c|c}
\hline Name & Abbreviation & Charge & Producer/company \\
\hline Cholesterol & $\mathrm{CHO}$ & No charge & Aldrich \\
\hline $\begin{array}{c}\text { Phosphatidylcholine } \\
\text { (soybean lecithin) }\end{array}$ & $\mathrm{SL}$ & $+/-$ & Aldrich \\
\hline
\end{tabular}




\section{3) Chemicals and reagents used}

All chemicals and reagents were high purity grade (〈Table 3$\rangle$ ).

\section{Preparation of Liposomes}

Liposomes were prepared by Reverse phase evaporation method. Each material was accurately weighed and placed into two separate vessels, one for the aqueous phase and one for the organic phase (chloroform). The two phases were mixed together to form water in oil (w/o) emulsion (Reverse micelles). The organic solvent was evaporated to form a gel, the reverse micelles then collapses to form a suspension. The suspension is re-dissolved a second organic solvent (methanol) and the solvent is evaporated under reduced pressure to form liposome. The volume is finally readjusted with water.

\section{RESULTS AND DISCUSSIONS}

\section{Roselle Extract}

〈Figure 6〉 shows the physical appearances of Roselle calyces and grinded

〈Table 3〉 Showing chemicals and reagents used in the experiments

\begin{tabular}{|c|c|c|}
\hline Name & Abbreviation & Producer/company \\
\hline Chloroform & CFM & Daejung Chemical Ltd. \\
\hline Ethyl acetate & EA & Aldrich \\
\hline Ethyl alcohol (ethanol) & $\mathrm{ETOH}$ & Samchun Chemicals Ltd. \\
\hline Folin ciocalteau phenol reagent & FCR & Aldrich \\
\hline 2,2Diphenyl-2-picrylhydrazyl & DPPH & Samchun Chemicals Ltd. \\
\hline Gallic acid & GA & Aldrich \\
\hline Methanol & $\mathrm{MTOH}$ & Samchun Chemicals Ltd. \\
\hline Orthophosphoric acid & OPA & Aldrich \\
\hline Sodium carbonate & $\mathrm{Na}_{2} \mathrm{CO}_{3}$ & Samchun Chemicals Ltd. \\
\hline Tween 80 & T.80 & Allshinwells co. Ltd. \\
\hline
\end{tabular}




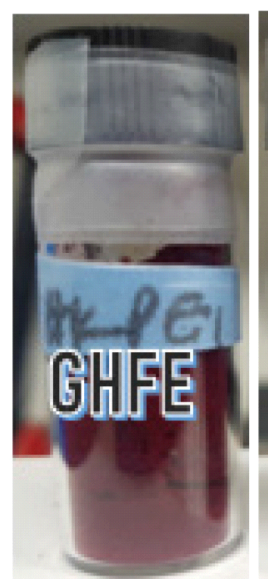

(a)

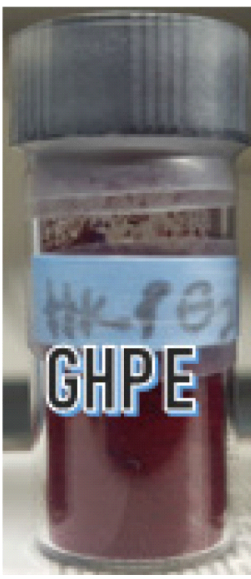

(b)

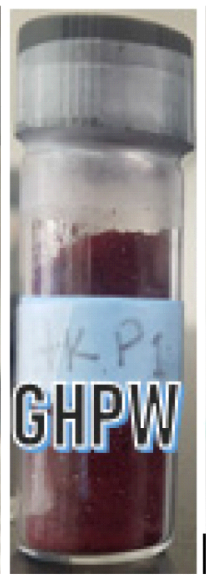

(c)

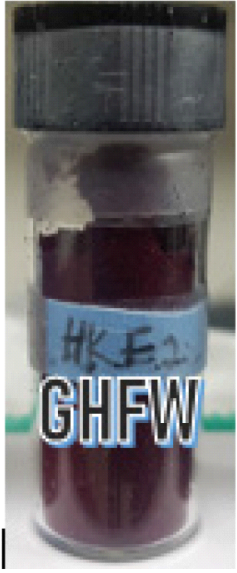

(d)

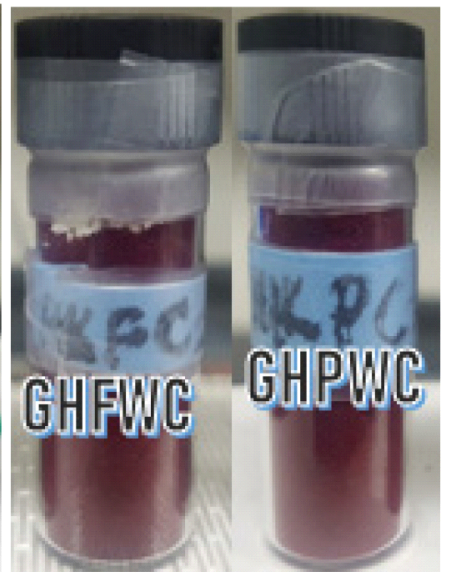

(e)

(f)

calyces extracted with different solvents. They are labelled as follows: (a) ethanolic extraction of whole Roselle calyces (GHFE), (b) ethanolic extraction of grinded Roselle calyces (GHPE), (c) water extraction of grinded Roselle calyces (GHPW), (d) water extraction of whole Roselle calyces (GHFW), (e) water, ethyl alcohol and chloroform extraction of whole Roselle calyces (GHFWC) and water, ethyl alcohol and chloroform extraction of whole Roselle calyces (GHPWC). All extracts show bright red in appearance except for GHPW \& GHFW which shows dark red in color. The \% average yield of the extracts are as follows: GHFE $(49.97 \pm 1.4)$, GHPE $(52.88 \pm 8)$, GHPW $(51.49 \pm 1.5)$, GHFW (45.26 \pm 13.8$)$, GHFWC (52.42 \pm 4$)$, GHPWC (56.06 \pm 1.9$)$.

〈Figure 7〉 shows a bar graph of percentage average yield of Roselle extract, GHPWC has the highest yield with $56.06 \pm 1.9 \%$ whiles the water extraction of the whole calyces GHFW has the lowest yield with $45.26 \pm 13.8 \%$.

\section{Green Tea Extracts}

〈Figure 8〉 shows the physical appearances of whole green tea leaves and grinded green tea leaves extracted with different solvents. They are labelled as follows: (a) ethanolic extract from whole green tea leaves (GTLE), (b) ethanolic 
〈Figure 7〉 A graph showing the \% average yield of Roselle extraction

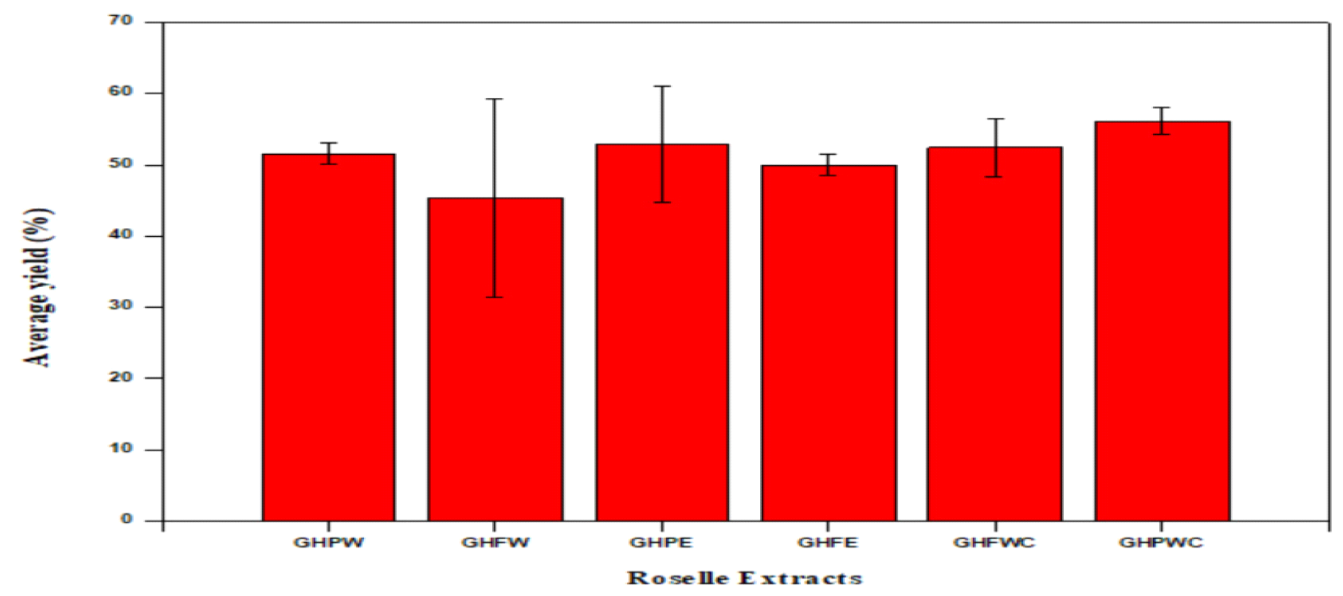

〈Figure 8〉 A figure showing green tea extracts

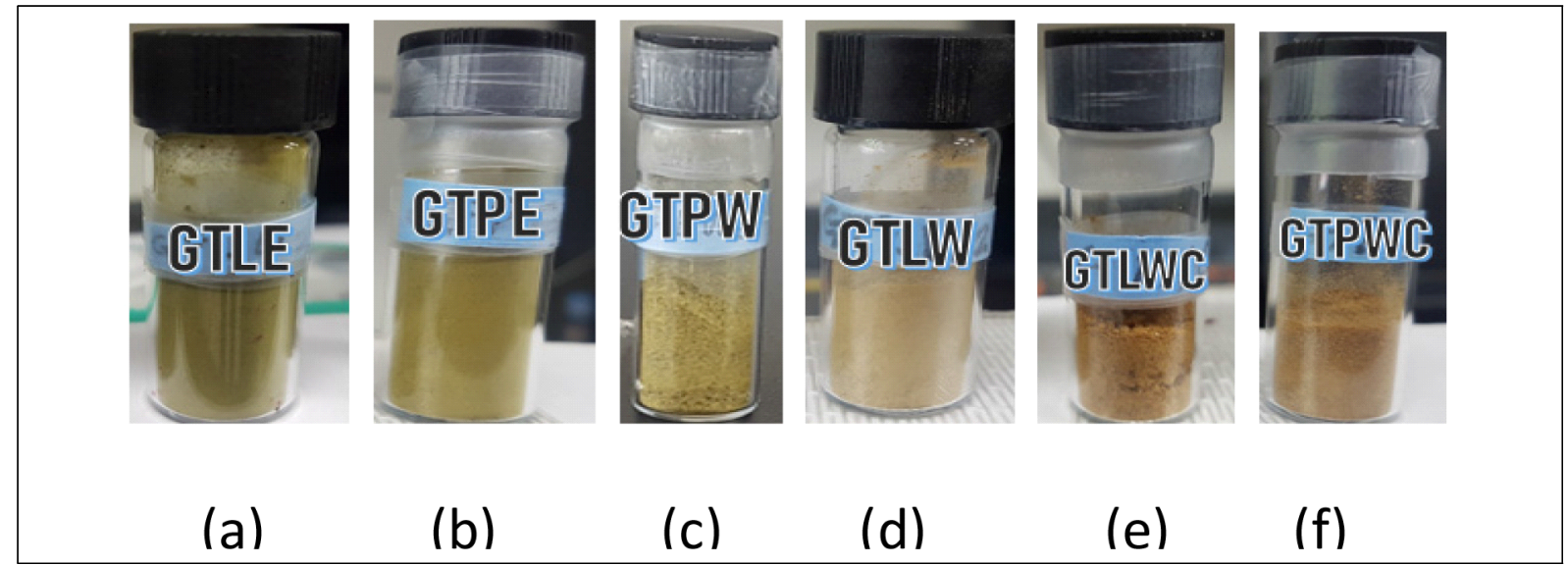

extract from grinded green tea leaves (GTPE), (c) water extract from grinded green tea leaves (GTPW), (d) water extract from whole green tea leaves (GTLW), (e) water, ethyl alcohol and chloroform extract from whole green tea leaves (GTLWC) and (f) water, ethyl alcohol and chloroform extract from grinded green tea leaves (GTPWC).

The GTLE \& GTPE are greenish with \% average yield of $33.66 \pm 0.23 \& 34.86$ \pm 3.17. The GTPW \& GTLW are light green in appearance with \% average yield of $27.83 \pm 1.38 \& 30.35 \pm 0.9$.

GTLWC \& GTPWC extracts shows brownish green in appearance with \% average yield of $19.22 \pm 1.20 \& 19.42 \pm 2.28$ respectively.

〈Figure 9〉 shows a bar graph of percentage average yield of Green tea, GTPE 
〈Figure 9〉\% average yield of green tea

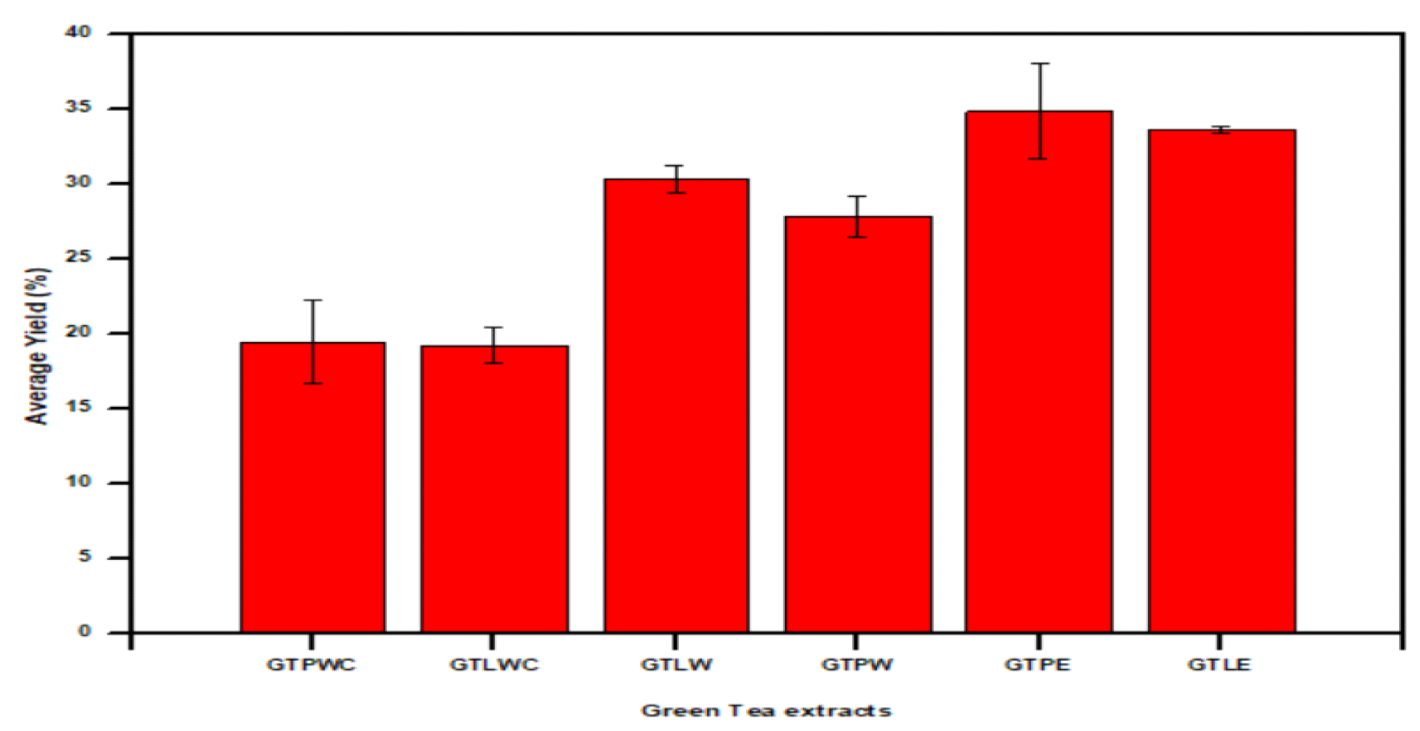

has the highest yield with $34.86 \pm 3.17 \%$ whiles GTLWC has the lowest yield with $19.22 \pm 1.19 \%$.

〈Figure 10〉 shows a bar graph of TPC of Roselle extracts from different solvents. TPC of the various Roselle extracts are as follows: GHPW (54.57 \pm 0.11 mgGAE/g), GHFW (60.85 $\pm 0.21 \mathrm{mgGAE} / \mathrm{g})$, GHPE (60.38 $\pm 0.21 \mathrm{mgGAE} / \mathrm{g})$, GHFE (53.78 $\pm 0.17 \mathrm{mgGAE} / \mathrm{g})$, GHFWC (45.49 $\pm 0.34 \mathrm{mgGAE} / \mathrm{g})$, GHPWC (34.89 $\pm 0.34 \mathrm{mgGAE} / \mathrm{g})$.

GHFW has the highest bar with TPC of $60.85 \pm 0.21$ whiles GHPWC has the lowest bar with $34.89 \pm 0.34 \mathrm{mgGAE} / \mathrm{g}$.

〈Figure 10〉 Total phenolic content of Roselle in different solvents

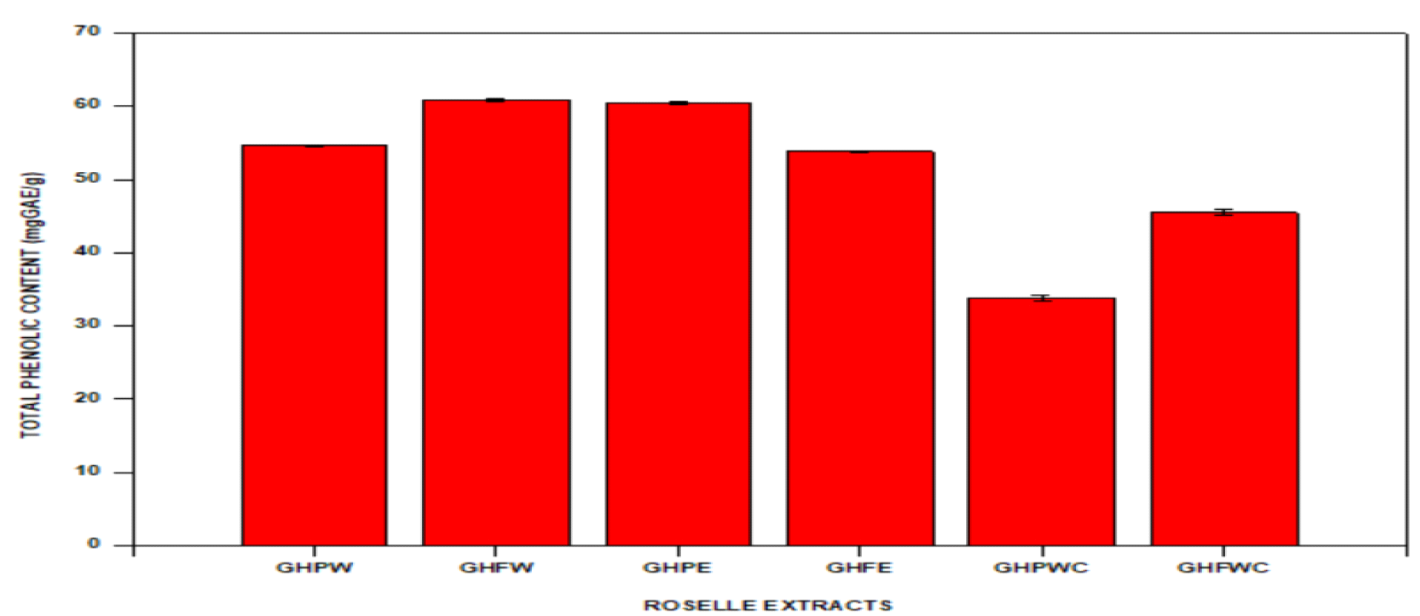


〈Figure 11〉 shows a bar graph of TPC of Green tea extracts from different solvents. TPC of the various Green tea extracts are as follows: GTPWC (179.63 $\pm 0.81 \mathrm{mgGAE} / \mathrm{g})$, GTLWC (302.49 $\pm 0.17 \mathrm{mgGAE} / \mathrm{g})$, GTLW (332.73 \pm 0.13 mgGAE/g), GTPW (280.55 $\pm 0.15 \mathrm{mgGAE} / \mathrm{g})$, GTPE (248.58 $\pm 0.15 \mathrm{mgGAE} / \mathrm{g})$, GTLE $(237.73 \pm 1.04 \mathrm{mgGAE} / \mathrm{g})$.

GTLW has the highest bar with TPC of $332.73 \pm 0.13 \mathrm{mgGAE} / \mathrm{g}$ whiles GTPWC has the lowest bar with TPC of $179.63 \pm 0.81 \mathrm{mgGAE} / \mathrm{g}$.

All extracts of Roselle show bright red in appearance except for GHPW \& GHFW which shows dark red in color. GHPWC has the most abundant yield with $56.06 \pm 1.9 \%$ whiles GHFW has the lowest yield with $45.26 \pm 13.8 \%$.

The GTLE \& GTPE are greenish with \% average yield of $33.66 \pm 0.23 \& 34.86$ \pm 3.17. The GTPW \& GTLW are light green in appearance with $\%$ average yield of $27.83 \pm 1.38 \& 30.35 \pm 0.9$. GTLWC \& GTPWC extracts shows brownish green in appearance with \% average yield of $19.22 \pm 1.20 \& 19.42 \pm 2.28$ respectively.

GHFW has the highest bar with TPC of $60.85 \pm 0.21$ whiles GHPWC has the lowest bar with $34.89 \pm 0.34 \mathrm{mgGAE} / \mathrm{g}$.

GTLW has the highest bar with TPC of $332.73 \pm 0.13$ mgGAE/g whiles GTPWC has the lowest bar with TPC of $179.63 \pm 0.81 \mathrm{mgGAE} / \mathrm{g}$.

TPCs of Roselle extracts from different solvents were found to be GHPW; 54.58 $\pm 0.01 \mathrm{mgGAE} / \mathrm{g}$, GHFW; $60.85 \pm 0.04 \mathrm{mgGAE} / \mathrm{g}$, GHPWC; $34.89 \pm 0.05 \mathrm{mgGAE} / \mathrm{g}$,

〈Figure 11〉 Total phenolic content of green tea extracts in different solvents

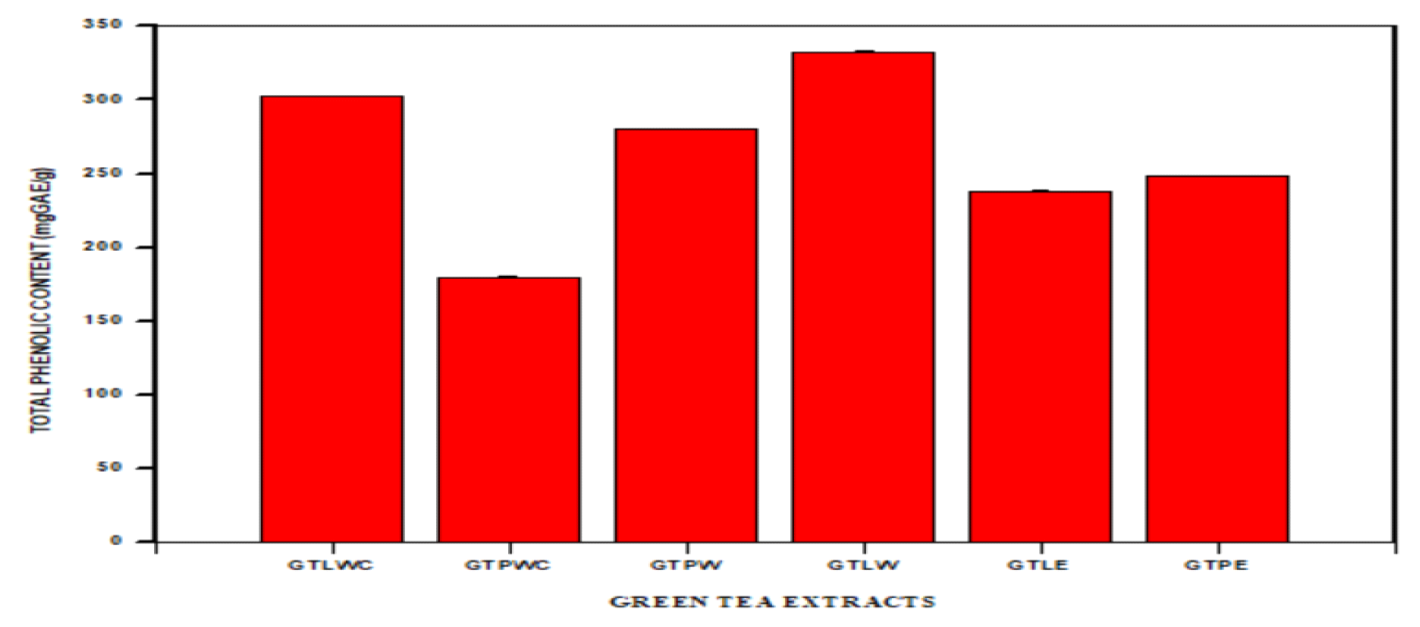


GHFWC; $45.50 \pm 0.06$ mgGAE/g, GHFE; $53.78 \pm 0.17$ mgGAE/g, GHPE; $60.39 \pm$ $0.08 \mathrm{mgGAE} / \mathrm{g}$. These values are higher than previously reported results of total polyphenolic contents of Hibiscus sabdariffa calyx by (Anokwuru et al., 2011). Results evidenced that extract yielded 29., 27.6, 19.3, and $27.6 \mathrm{mg} \mathrm{GAE} \mathrm{g}^{-1}$ respectively with methanol, ethanol, acetone and water as solvents. (Dalar et al., 2012) reported maximum levels of total phenols of $17.4 \pm 0.3 \mathrm{mg} \mathrm{GAE} \mathrm{g}^{-1}$ of dry weight of the lyophilized powder and $35.3 \pm 2.8 \mathrm{mg} \mathrm{GAE}^{-1}$ of dry weight of the lyophilized powder in acidified methanolic extracts of Malva neglecta and Plantago lanceolata, respectively. TPCs were however similsr to the results obtained by (Medina et al., 2011) studies on the effect and activity of araca extracts as antioxidant, antimicrobial and anti-proliferative features on human cancer cells. (Wootton-Beard et al., 2011) investigated total polyphenol content of 23 commercially available vegetable juices before and after in vitro digestion.

In general, the results obtained in current assays indicated that the extraction of total polyphenols contents was maximized and the compounds were superior to other plants reported in the literature. Bars represent the means of experiments carried out in triplicates.

Statistical Analysis. Data were expressed as the means (standard error of the means of the experiments carried out in triplicates. A one-way ANOVA single factor was used to evaluate the significance of results. A probability $(p)$ value $<0.05$ was considered significant.

\section{Empty Liposome}

〈Figure 12〉 shows the physical appearances of empty liposome formulations as follows:

a is empty liposome containing only cholesterol with lipid concentrations as $80 \mu \mathrm{mole} / \mathrm{mL}$ (ACT80E).

$\mathrm{b}$ is empty liposome with lipid concentrations as $80 \mu \mathrm{mole} / \mathrm{mL}$ (AST80E).

$\mathrm{c}$ is empty liposome containing cholesterol with lipid concentrations as 80 $\mu \mathrm{mole} / \mathrm{mL}$ (ACTS80E). All formulations gave milky to yellowish colloidal appearance depending on the amount of total lipid content in formulations. The liposome with lowest total lipid content exhibited the milky colloidal appea- 
〈Figure 12〉 Physical appearances and morphology of empty liposomes by phase contrast microscope

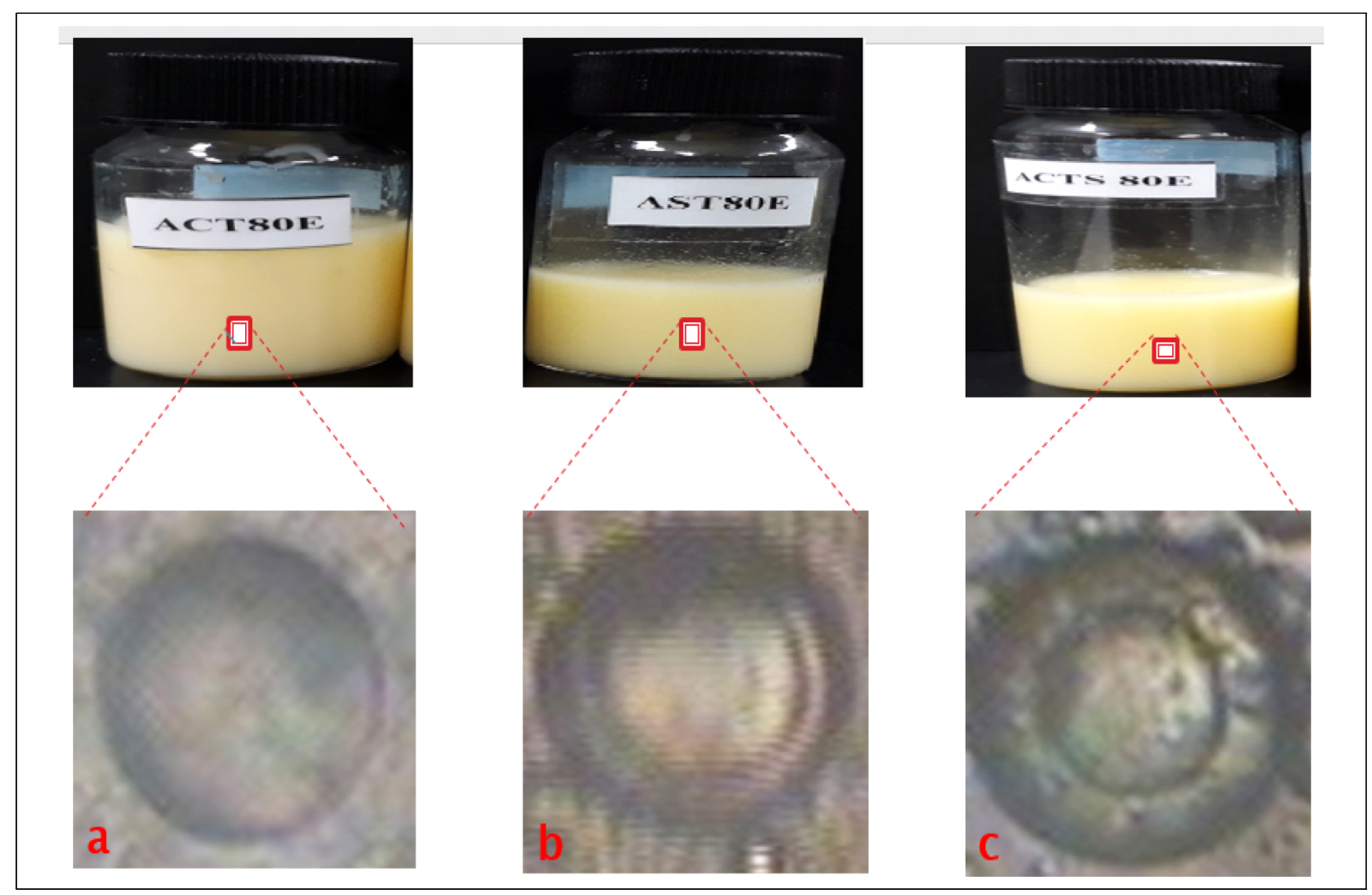

rances. Also, the formulations with the highest total lipid content showed the yellowish colloidal appearances. No precipitation and phase separation were observed in day 1.

〈Figure 12〉 shows the morphology of empty liposome formulation using phase contrast microscope. a is ACT80E has a thinner shell/wall with large aqueous core, $\mathrm{b}$ is AST80E which indicates a large aqueous core with slightly thicker and larger wall, $\mathrm{c}$ is ACTS80E and the image forms has thicker and larger wall/shell with slightly bigger aqueous core.

\section{Green Tea Loaded Liposomes}

〈Figure 13〉 shows the physical appearances of all Green tea loaded liposome formulations as follows:

a is empty liposomes containing cholesterol and green tea extract with lipid concentrations of $80 \mu \mathrm{mole} / \mathrm{mL}$ (ACT80GT). b is empty liposomes containing 
〈Figure 13〉 Physical appearances and morphology of green tea loaded liposomes by phase contrast microscope

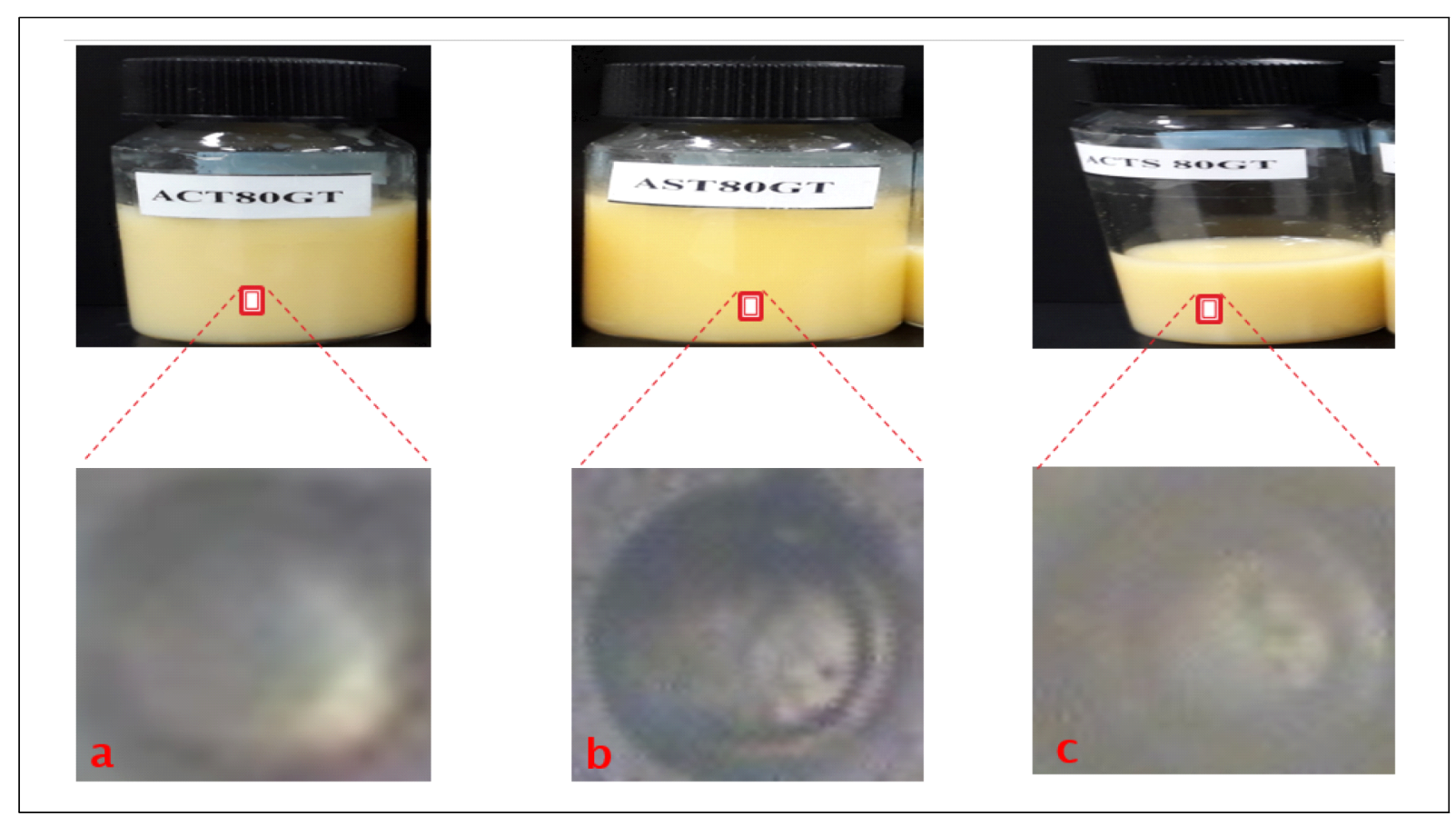

Green tea extract with lipid concentrations of $80 \mu \mathrm{mole} / \mathrm{mL}$ (AST80GT). c is empty liposomes containing cholesterol and Green tea with lipid concentration of $80 \mu \mathrm{mole} / \mathrm{mL}$ (ACTS80GT).

The physical appearances of all the Green tea loaded liposomes formulations gave milky, slightly yellowish to yellowish colloidal appearance depending on the amount of total lipid content in formulations. The Green tea loaded liposome with cholesterol and the lowest total lipid content exhibited the milky colloidal appearances. The formulation without cholesterol but with lowest lipid content shows slightly yellowish colloidal appearances. Also, the formulations with the highest total lipid content showed the yellowish colloidal appearances as shown in the formulations ACT80GT, AST80GT, and ACTS80GT. No precipitation and phase separation were observed in day 1.

〈Figure 12〉 shows the morphology of Green tea loaded liposome formulation with concentration of $80 \mu \mathrm{mol} / \mathrm{mL}$ using phase contrast microscope. a is (ACT80GT) and has thinner shell/wall with large aqueous core, $b$ is (AST80GT), which indicates a large aqueous core with slightly thicker and larger wall, c is (ACTS80GT) and the image forms has thicker and larger wall/shell with slightly 


\section{Roselle Loaded Liposome}

〈Figure 14〉 shows the physical appearances of Roselle loaded liposome formulations as follows: $\mathbf{a}$ is empty liposomes containing cholesterol and Roselle extract with lipid concentration of $80 \mu \mathrm{mol} / \mathrm{mL}$ (ACT80HB). b is empty liposomes containing Roselle extract with lipid concentration of $80 \mu \mathrm{mol} / \mathrm{mL}$ (AST80HB). c is empty liposomes containing cholesterol and Roselle extract with different lipid concentration of $80 \mu \mathrm{mol} / \mathrm{mL}$ (ACTS80HB).

The physical appearances of all Roselle liposome formulations gave pinkish to yellowish colloidal appearance depending on the amount of total lipid content in formulations. The Liposome with cholesterol and the lowest total lipid content exhibited pinkish colloidal appearances as shown in the formulations.

〈Figure 14〉 Physical appearances and morphology of Roselle extract loaded liposomes using phase contrast microscope

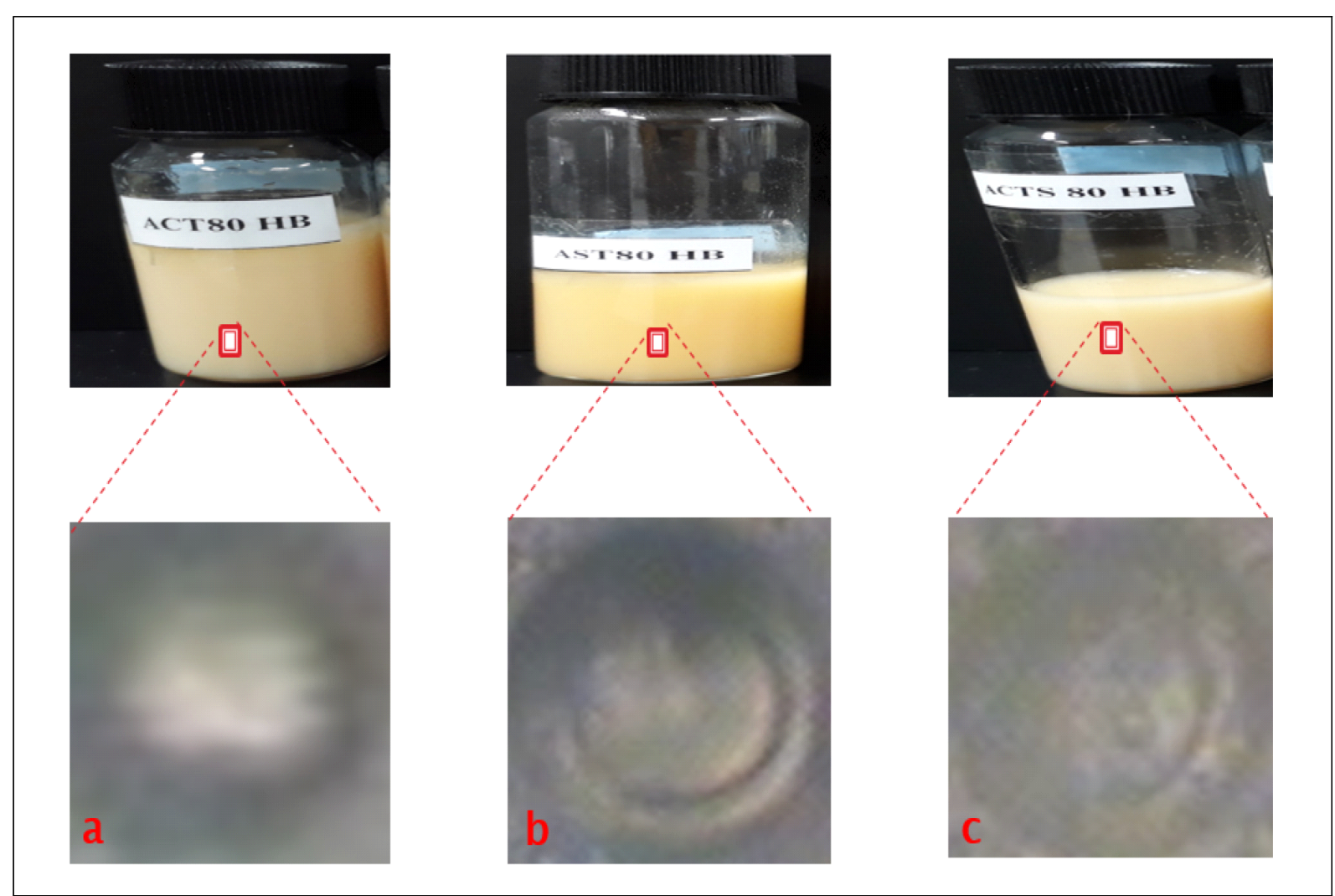


The rest of the formulations showed slightly yellowish or yellowish colloidal appearances as in AST80HB and ACTS80HB. No precipitation and phase separation were observed in day 1 .

〈Figure 14〉 shows the morphology of Roselle loaded liposome formulation with concentration of $80 \mu \mathrm{mol} / \mathrm{mL}$ using phase contrast microscope. a is (ACT80HB) and has thinner shell/wall with large aqueous core, b is (AST80HB), and c (ACTS80HB) indicate large aqueous core with slightly thicker and larger wall.

\section{Empty Liposomes Containing Cholesterol with Either Green Tea or Roselle}

〈Figure 15〉 shows the physical appearances of liposome formulations as follows: a is empty liposomes containing only cholesterol with lipid concentration of $80 \mu \mathrm{mol} / \mathrm{mL}$ (ACT80E). b is empty liposomes containing cholesterol and Green tea with lipid concentration of $80 \mu \mathrm{mol} / \mathrm{mL}$ (ACT8GT). c is empty liposomes containing cholesterol and Roselle extract with lipid concentration of 80 $\mu \mathrm{mol} / \mathrm{mL}$ (ACT80HB). 〈Figure 15〉 shows the morphology of different liposome formulations with concentration of $80 \mu \mathrm{mol} / \mathrm{mL}$ using phase contrast microscope. All the three a (ACT80E), b (ACT80GT), and c (ACT80HB) have thinner shell/wall with large aqueous core.

〈Figure 15〉 Empty, green tea and Roselle loaded liposomes with only cholesterol

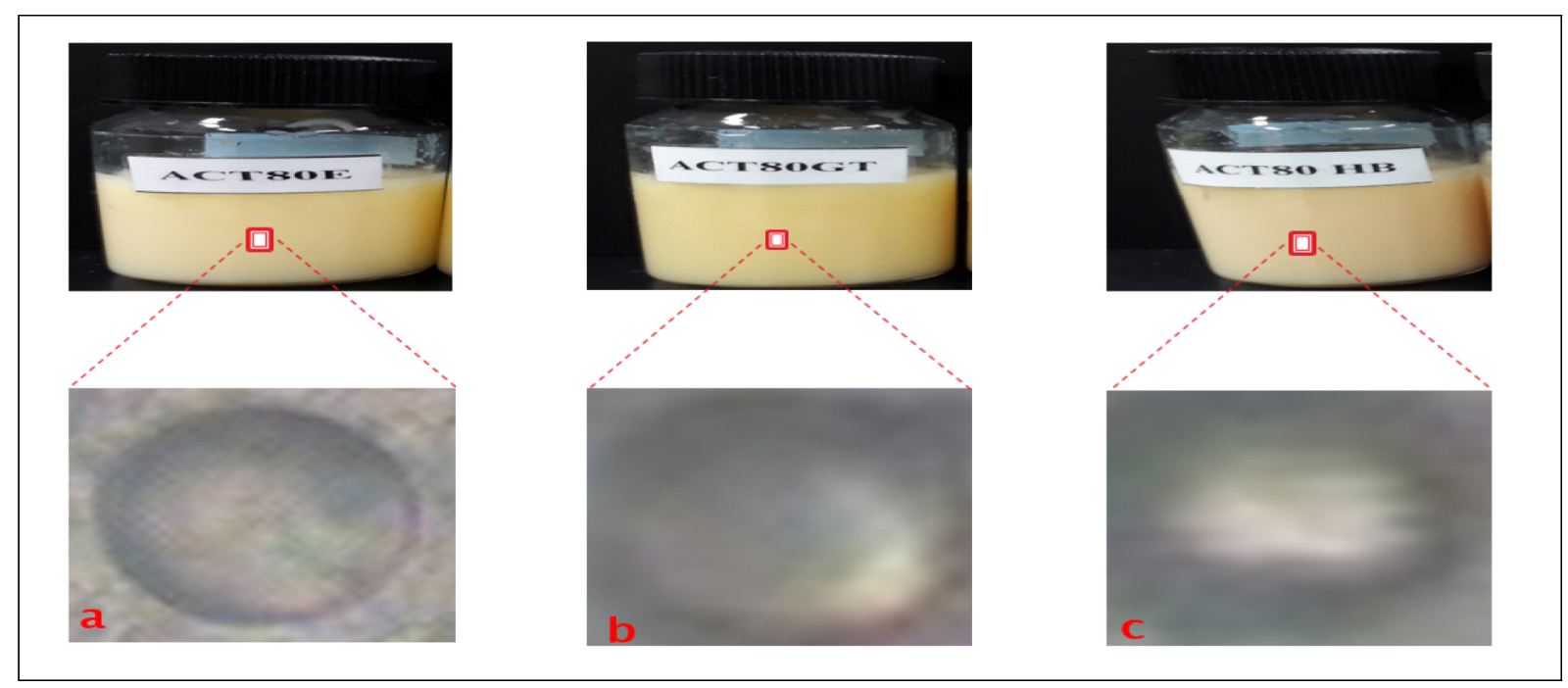


〈Figure 16〉 shows the morphology of different liposome formulations with concentration of $80 \mu \mathrm{mol} / \mathrm{mL}$ using phase contrast microscope. All the three a (ACTS80E), b (ACTS80GT), and c (ACTS80HB) have large and thicker shell/wall with slightly larger aqueous core.

Size and size distribution (polydispersity) of the formulated liposomes are of particular importance in their characterization. Maintaining a constant size and/or size distribution for a prolonged period of time is an indication of liposome stability.

In this experiment microscopic methods and dynamic light scattering were used for establishing the morphology, size and stability of liposome.

The changes in the morphology of liposomes obtained by natural swelling in the presence of cholesterol. The mean particle size of green tea and Roselle extract loaded liposomes prepared by reverse phase evaporation method was measured to investigate the stability of the systems. Previous studies indicate the mean particle size was highly dependent on the composition of liposomes. In the study carried by (Gibis et al., 2012) it was concluded the size of the liposomes depended on the material that was encapsulated into liposomes.

The higher mean particle size of the extract loaded liposomes result might be explained by the fact that phenolic compounds might be incorporated into a lipid bilayer and/or may be absorbed onto the surface of liposomes as well as incorporated into the interior region of the liposomes which could be due to

〈Figure 16〉 Empty, green tea and Roselle loaded liposomes with both cholesterol and squalene

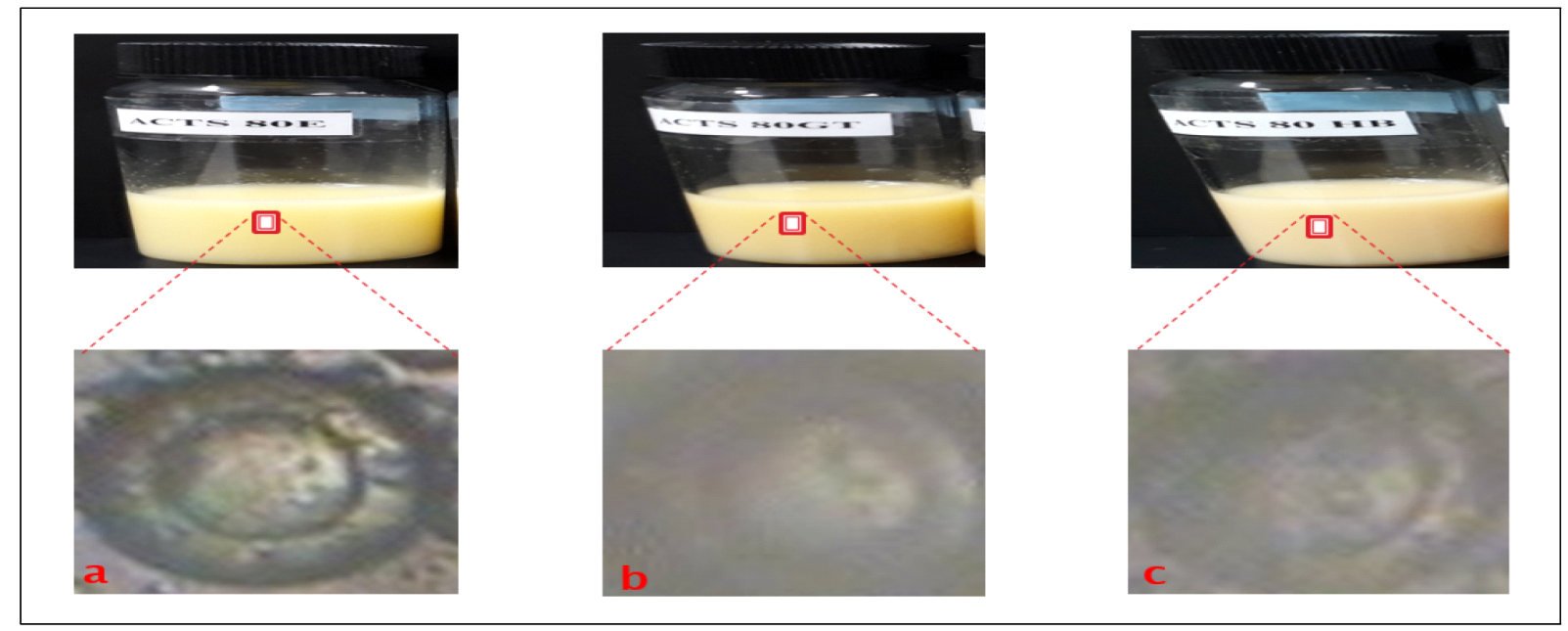


hydrogen bonding between polar head groups and the phenolic compounds in the extract, hydrophobic interactions between the fatty acid tail so of the polar lipid and the more hydrophobic moieties of the phenolic compounds, or thermodynamic driving forces such as the liposomes attaining a more optimal configuration (Gibis et al., 2012).

\section{CONCLUSION}

In conclusion, Green tea and Roselle extracts are natural antioxidants rich in polyphenols. The role of polyphenols from Green tea and Roselle extracts have great deal of potential as part of a growing natural antiaging and whitening skin care market with all-natural ingredients however, it is important to standardize extraction methods as well as their assay for their activity.

In the scope of this study, the Roselle and the green tea extract were encapsulated into liposomes by dispersing soy lecithin in distilled water by Reverse phase evaporation method in order to enhance the stability of polyphenols and protect their functional properties.

It was found that the Liposomes produced with only cholesterol gave a larger aqueous core with thinner wall/shell and most stable formulations. The formulations with Cholesterol gave the smallest aqueous core but thicker wall/shell.

The mean particle size of the extract loaded liposomes were high in almost all cases than the empty liposomes.

The higher mean particle size of the extract loaded liposomes might be explained by the fact that phenolic compound might be incorporated into lipid bilayer and/or may be absorbed onto the surface of liposome due to hydrogen bonding between polar head groups and the phenolic compounds in the extract (Gibis et al., 2012).

\section{REFERENCES}

Akbarzadeh, A., Rezaei-Sadabady, R., Davaran, S., Joo, S. W., Zarghami, N., Hanifehpour, Y., Samiei, M., Kouhi, M., \& Nejati-Koshki, K. (2013). Liposome: 
classification, preparation, and applications. Nanoscale Research Letters, 8(1), 102.

Anokwuru, C. P., Esiaba, I., Ajibaye, O., \& Adesuyi, A. O. (2011). Polyphenolic content and antioxidant activity of Hibiscus sabdariffa calyx. Research Journal of Medicinal Plant, 5(5), 557-566.

Babu, PV., \& Liu, D. (2008). Green tea catechins and cardiovascular health: An update. Current Medicinal Chemistry, 15(18), 1840-1850.

Bergmeier, D., Berres, P. H. D., Filippi, D., Bilibio, D., Bettiol, V. R., \& Priamo, W. L. (2014). Extraction of total polyphenols from hibiscus (Hibiscus sabdariffa L.) and waxweed/'sete-sangrias' (Cuphea carthagenensis) and evaluation of their antioxidant potential. Acta Scientiarum. Technology, 36(3), 545-551.

Betz, G., Aeppli, A., Menshutina, N., \& Leuenberger, H. (2005). In vivo comparison of various liposome formulations for cosmetic application. International Journal of Pharmaceutics, 296(1-2), 44-54.

Bibi, S., Kaur, R., Henriksen-Lacey, M., McNeil, S. E., Wilkhu, J., Lattmann, E., Christensen, D., Mohammed, A. R., \& Perrie, Y. (2011). Microscopy imaging of liposomes: from coverslips to environmental SEM. International Journal of Pharmaceutics, 417(1-2), 138-150.

Blume, G., Sacher, M., Teichmuller, D., \& Schafer, U. (2003). The role of liposomes and their future perspective. SOFW Journal, 129(8), 10-14.

Briuglia, M. L., Rotella, C., McFarlane, A., \& Lamprou, D. A. (2015). Influence of cholesterol on liposome stability and on in vitro drug release. Drug Delivery and Translational Research, 5(3), 231-242.

Cevc, G., \& Blume, G. (1992). Lipid vesicles penetrate into intact skin owing to the transdermal osmotic gradients and hydration force. Biochimica et Biophysica Acta (BBA)-Biomembranes, 1104(1), 226-232.

Choi, M. J., \& Maibach, H. I. (2005). Liposomes and niosomes as topical drug delivery systems. Skin Pharmacology and Physiology, 18(5), 209-219.

Choudhury, B. (2013). Development of giant liposomal formulation for drug delivery and tissue engineering application. (Bachelor's Thesis). National Institute of Technology, Rourkela, India.

Chrai, S. S., Murari, R., \& Ahmad, I. (2002). Liposomes: A review. Pharmaceutical Technology, 26(4), 28-34. 
Dag, D., \& Oztop, M. H. (2017). Formation and characterization of green tea extract loaded liposomes. Journal of Food Science, 82(2), 463-470.

Dalar, A., Turker, M., \& Konczak, I. (2012). Antioxidant capacity and phenolic constituents of Malva neglecta Wallr. and Plantago lanceolata L. from Eastern Anatolia region of Turkey. Journal of Herbal Medicine, 2(2), 42-51.

Daniele, B., Berres, P. H. D., Filippi, D., Bilibio, D., Bettiol, V. R., \& Priamo, W. L. (2014). Extraction of total polyphenols from hibiscus (Hibiscus sabdariffa L.) and waxweed / 'sete-sangrias' (Cuphea carthagenensis) and evaluation of their antioxidant potential. Acta Scientiarum. Technology, 36(3), 545-551.

Dragicevic, N., \& Maibach, H. I. (2016). Percutaneous penetration enhancers chemical methods in penetration enhancement. Berlin, German: Springer.

Dua, J. S., Rana, A. C., \& Bhandari, A. K. (2012). Liposome: methods of preparation and applications. International Journal of Pharmaceutical Studies and Research, 3(2), 14-20.

Edwards, K. A. (2016). Liposomes in analytical methodologies. (pp. 1-82). Boca Raton, FL: Pan Standard Publishing.

Fakhravar, Z., Ebrahimnejad, P., Daraee, H., \& Akbarzadeh, A. (2016). Nanoliposomes: Synthesis methods and applications in cosmetics. Journal of Cosmetic and Laser Therapy, 18(3), 174-181.

Gibis, M., Vogt, E., \& Weiss, J. (2012). Encapsulation of polyphenolic grape seed extract in polymer-coated liposomes. Food \& Function, 3(3), 246-254.

Grumezescu, A. M. (2018). Organic materials as smart nanocarriers for drug delivery. William Andrew.

Hjorth, N. (1965). Contact sensitivity to plants and balsams. Paper presented at the Symposium on Allergic Contact Eczema in Theory and Practice (Europ Congr Allergy).

Ho, N. F. H., Ganesan, M. G., Weiner, N. D., \& Flynn, G. L. (1985). Mechanisms of topical delivery of liposomally entrapped drugs. Journal of Controlled Release, 2, 61-65.

Horie, H., \& Kohata, K. (2000). Analysis of tea components by high-performance liquid chromatography and high-performance capillary electrophoresis. Journal of Chromatography A, 881(1-2), 425-438.

Knepp, V. M., Szoka, F. C., \& Guy, R. H. (1990). Controlled drug release from a 
novel liposomal delivery system. II. Transdermal delivery characteristics. Journal of Controlled Release, 12(1), 25-30.

Laouini, A., Jaafar-Maalej, C., Limayem-Blouza, I., Sfar, S., Charcosset, C., \& Fessi, H. (2012). Preparation, characterization and applications of liposomes: state of the art. Journal of Colloid Science and Biotechnology, 1(2), 147-168. Lasch, J., \& Wohlrab, W. (1986). Liposome-bound cortisol: a new approach to cutaneous therapy. Biomedica Biochimica Acta, 45(10), 1295-1299.

Lautenschlager, H. (2006). Liposomes. In A. O. Barel, M. Paye \& H. I. Maibach (Eds.), Handbook of cosmetic science and technology (2nd ed., pp. 155-163). Boca Raton: CRC Press.

Li, Z., Paulson, A. T., \& Gill, T. A. (2015). Encapsulation of bioactive salmon protein hydrolysates with chitosan-coated liposomes. Journal of Functional Foods, 19, 733-743.

Lu, Z. (2014). Evaluation of encapsulation process for improved loading of lysozyme in liposomes. (Master's thesis). Central University of China, Beijing, China.

Medina, A. L., Haas, L. I. R., Chaves, F. C., Salvador, M., Zambiazi, R. C., Da Silva, W. P., Nora, L., \& Rombaldi, C. V. (2011). Araçá (Psidium cattleianum Sabine) fruit extracts with antioxidant and antimicrobial activities and antiproliferative effect on human cancer cells. Food Chemistry, 128(4), 916-922.

Muller-Goymann, C. (2004). Physicochemical characterization of colloidal drug delivery systems such as reverse micelles, vesicles, liquid crystals and nanoparticles for topical administration. European Journal of Pharmaceutics and Biopharmaceutics, 58(2), 343-356.

New, R. (1990). Preparation of liposomes and size determination. In New RRC (Ed.), Liposomes: A practical approach (pp. 36-39). Oxford, UK: Oxford University Press.

Patravale, V. B., \& Mandawgade, S. D. (2008). Novel cosmetic delivery systems: an application update. International Journal of Cosmetic Science, 30(1), 19-33.

Pinsuwan, S., Amnuaikit, T., Ungphaiboon, S., \& Itharat, A. (2010). Liposomecontaining Hibiscus sabdariffa calyx extract formulations with increased antioxidant activity, improved dermal penetration and reduced dermal toxicity. Journal of the Medical Association of Thailand= Chotmaihet Thangphaet, 93, 
S216-S226.

Sindhi, V., Gupta, V., Sharma, K., Bhatnagar, S., Kumari, R., \& Dhaka, N. (2013). Potential applications of antioxidants: A review. Journal of Pharmacy Research, 79), 828-835.

Singh, A., Vengurlekar, P., \& Rathod, S. (2014). Design, development and characterization of liposomal neem gel. International Journal of Pharma Science Research, 5, 140-148.

Sulkowski, W. W., Pentak, D., Nowak, K., \& Sulkowska, A. (2005). The influence of temperature, cholesterol content and $\mathrm{pH}$ on liposome stability. Journal of Molecular Structure, 744-747, 737-747.

Tejeda-Mansir, A., Garcia-Rendon, A., \& Guerrero-German, P. (2019). Plasmid-DNA lipid and polymeric nanovaccines: a new strategic in vaccines development. Biotechnology and Genetic Engineering Reviews, 35(1), 46-68.

Verma, D. D., Verma, S., Blume, G., \& Fahr, A. (2003). Particle size of liposomes influences dermal delivery of substances into skin. International Journal of Pharmaceutics, 258(1-2), 141-151.

Wootton-Beard, P. C., Moran, A., \& Ryan, L. (2011). Stability of the total antioxidant capacity and total polyphenol content of 23 commercially available vegetable juices before and after in vitro digestion measured by FRAP, DPPH, ABTS and Folin-Ciocalteu methods. Food Research International, 44(1), 217224.

Zhang, H. (2017). Thin-film hydration followed by extrusion method for liposome preparation. In G. G. M. D'Souza (Ed.), Liposomes (pp. 17-22). New York, NY: Springer. 